\title{
2D/3D Registration for Measurement of Implant Alignment After Total Hip Replacement
}

\author{
Branislav Jaramaz ${ }^{1,2}$ and Kort Eckman ${ }^{2}$ \\ ${ }^{1}$ The Western Pennsylvania Hospital, Pittsburgh, Pennsylvania, USA \\ ${ }^{2}$ Carnegie Mellon University, Pittsburgh, Pennsylvania, USA \\ branko@icaos.org, korteandrew.cmu.edu
}

\begin{abstract}
Measurements of cup alignment after total hip replacement (THR) surgery are typically performed on postoperative pelvic radiographs. Radiographic measurement of cup orientation depends on the position and orientation of the pelvis on the X-ray table, and its variability could introduce significant measurement errors. We have developed a tool to accurately measure 3D implant orientation from postoperative antero-posterior radiographs by registering to preoperative CT scans. The purpose of this study is to experimentally and clinically validate the automatic CT/X-ray matching algorithm by comparing the X-ray based measurements of cup orientation with direct 3D measurements from postoperative CT scans. The mean measurement errors ( \pm stdev) found in this study were $0.4^{\circ} \pm 0.8^{\circ}$ for abduction and $0.6^{\circ} \pm 0.8^{\circ}$ for version. In addition, radiographic pelvic orientation measurements demonstrated a wide range of inter-subject variability, with pelvic flexion ranging from $-5.9^{\circ}$ to $11.2^{\circ}$.
\end{abstract}

Keywords: radiograph, registration, mutual information, hip replacement, THR, THA.

\section{Introduction}

The standard postoperative follow-up procedure for total hip arthroplasty involves the assessment of implant orientation from antero-posterior (AP) radiographs to assure the surgeon that the implant is correctly aligned. Improper placement can lead to impingement and dislocation problems, increased polyethylene wear, and implant loosening. The long-term follow-up procedure typically involves obtaining a series of radiographs over a period of years to detect any changes in implant alignment or in surrounding bony tissue. Accurate implant orientation measurements provide useful feedback to the surgeon who, based on this information, may be able to detect a systematic bias in operative technique and adjust it accordingly. Information about implant orientation is also used to determine the need for revision surgery and other additional interventions. The standard postoperative follow-up procedure involves the acquisition of 2D AP radiographs. The orientation of the implant is then measured on the resultant radiograph. 
In the case of computer-assisted surgery, an accurate tool to measure postoperative implant orientation could serve to test overall system accuracy and the consistency of performance. Computer-assisted surgery typically provides the surgeon with the measurements of cup orientation (abduction and version) relative to the anterior pelvic plane (APP). This plane, defined by the anterior superior iliac spines and pubic tubercles provides a reliable and repeatable anatomic reference system for the pelvis. However, the same reference system cannot be established in AP radiographs, and some definition of the transverse axis, typically a line connecting the teardrops, is used instead. Consequently, the radiographic measurements of cup orientation may differ from those recorded intraoperatively. Furthermore, the accuracy of radiographic measurement depends on the position of the X-ray source relative to the film as well as on the orientation of the pelvis at the time the X-ray was taken. It is commonly assumed that for the AP radiograph the anterior pelvic plane is parallel to the film plane. Unfortunately, this is not guaranteed by the standard radiographic protocol. Pelvic flexion can vary considerably even for the same patient [3]. These pelvic pose variations will negatively impact implant measurement accuracy.

By co-registering a radiograph with both a synthetic X-ray, which is generated from a CT scan of the patient, and a projection of the implant CAD model, 2D/3D registration recovers the true pose of the implant with respect to the patient's anatomy. A single CT scan, preoperative or postoperative, can be used to analyze an entire series of X-rays. The purpose of the present study is to validate both experimentally and clinically a new CT/X-ray matching algorithm based on mutual information. During the past decade, mutual information has received considerable attention as an accurate registration tool. Nonetheless, mutual information usually contains many local maxima that affect the robustness of registration. Moreover, since mutual information also produces erroneous global maxima, its use is generally restricted to local registration tasks. Considerable research has been directed at improving the measure. Spatial information is simply ignored by direct application of mutual information as it is invariant to pixel/voxel reshuffling (excepting values that are the results of interpolation). Methods that modify mutual information and incorporate spatial information into the measure have demonstrated improved robustness. One of these methods is regional mutual information (RMI), which extends the dimensionality of the histograms [7]. Another method incorporates spatial information by multiplying conventional mutual information by a function that compares the gradient vectors of the images [6].

In this study, we used conventional mutual information and gradient comparisons along with pixel masking to exclude additional independent rigid objects (femurs) and prevent them from affecting the similarity measure. Erroneous maxima are avoided by coarse initial alignments of the implants and CT scans by the user. The validation is performed by comparing the measurements of cup orientation using $2 \mathrm{D} / 3 \mathrm{D}$ registration software, with direct $\mathrm{CT}$ measurements. The same clinical data were previously examined using interactive manual alignments of the synthetic and real radiographs [1]. In this study we report the results obtained with a fully automatic registration procedure. 


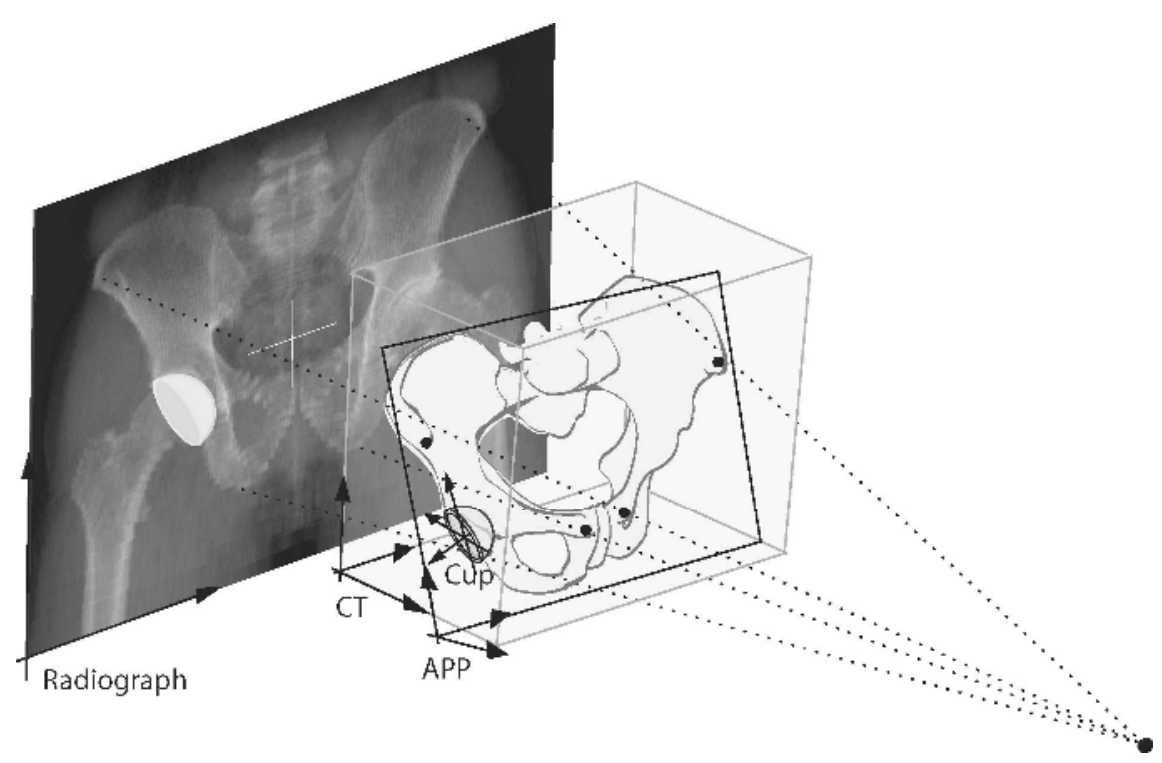

Fig. 1. Reference systems in the synthetic x-ray projection environment. The CT scan (CT) and the cup model (Cup) are placed relative to the x-ray film (Radiograph) in order to create a synthetic projection. The anatomic reference plane of the pelvis (APP) is specified in the CT scan.

\section{Materials and Method}

\subsection{D/3D Matching Protocol}

An original CT/ X-ray image-matching algorithm was developed to measure the pelvis orientation and acetabular cup position from AP radiographs using the following protocol:

1. An anatomic reference system for the pelvis ${ }^{\mathrm{CTpelvis}} \mathrm{T}_{\mathrm{APP}}$ is defined in the $\mathrm{CT}$ scan using the CT-based THR planning software. (T denotes a standard $4 x 4$ rigid transformation matrix)

2. A real pelvic AP radiograph was digitized (image A). All radiographs were taken using the standard 40" $(1016 \mathrm{~mm})$ source-to-film distance. The X-ray source locations were marked on the film using small metallic washers.

3. A synthetic pelvic AP radiograph is generated (image B) from the CT scan, with known (X-ray source position, film-to-source distance) and assumed (pose of the CT scan within the $\mathrm{X}$-ray system: ${ }^{\text {radiograph }} \mathrm{T}_{\mathrm{CTpelvis}}$ ) projection parameters (Fig 1.).

4. Image similarity was evaluated using a combination of mutual information [8], [9] and gradient comparisons [6]. For two images A and B, with a joint probability mass function $\mathrm{p}(\mathrm{a}, \mathrm{b})$ and marginal probability mass functions $\mathrm{p}(\mathrm{a})$ and $\mathrm{p}(\mathrm{b})$, the mutual information $\mathrm{I}(\mathrm{A} ; \mathrm{B})$ is defined as: 


$$
I(A ; B)=\sum_{a \in A} \sum_{b \in B} p(a, b) \log \frac{p(a, b)}{p(a) p(b)} .
$$

Mutual information can be rewritten using marginal and joint entropies in the following manner:

$$
I(A ; B)=H(A)+H(B)-H(A, B) .
$$

The two images (real and synthetic radiograph) are registered by maximizing the similarity function. In order to minimize the effects of additional rigid bodies, the femurs and implant components were masked within the procedure. The masked pixels were excluded from the gradient comparison and mutual information objective function. Gross initial alignments of the implants and CT scans were provided by the user.

5. The pose of the CT scan in the projection space was automatically manipulated until the best match between the real and the synthetic X-ray was found (Fig 2).

6. A virtual projection of the appropriate CAD implant model was generated for a given position and spatial orientation of the cup. The initial coarse alignment is specified by the user.

7. The cup position and orientation relative to the $\mathrm{x}$-ray film ${ }^{\text {radiograph }} \mathbf{T}_{\text {cup }}$ is iteratively modified using another MI based automatic method until the virtual projection best matches the projection of the cup in the real radiograph (Fig 3).

8. Cup orientation relative to the anatomic reference system of the pelvis APPTcup is calculated:

$$
{ }^{\mathrm{APP}} \mathrm{T}_{\text {cup }}={ }^{\mathrm{APP}} \mathrm{T}_{\mathrm{C} \text { (Tplvis }} \cdot{ }^{\mathrm{CTpelvis}} \mathrm{T}_{\text {radiograph }} \cdot{ }^{\text {radiograph }} \mathrm{T}_{\text {cup }} \cdot
$$

9. In addition, pelvic flexion ${ }^{\text {radiograph }} \mathrm{T}_{\mathrm{APP}}$ (inclination of the APP plane relative to the radiographic plane) is calculated from:

$$
{ }^{\text {radiograph }} \mathrm{T}_{\mathrm{APP}}={ }^{\text {radiograph }} \mathrm{T}_{\mathrm{CT} \text { pelvis }} \cdot{ }^{\mathrm{CTpelvis}} \mathrm{T}_{\mathrm{APP}} .
$$

Rendering synthetic radiographs from CT data sets can be a computationally intensive process. Moreover, registering real and synthetic radiographs requires the generation of numerous synthetic radiographs. In order to achieve registration in a timely manner, the radiographs were synthesized using custom shader programs running on special graphics hardware. Specifically, an nVidia GeForce 7800GT was used for Xray synthesis. This allowed for fast rendering of high dynamic range, floating-point precision images, and resulted in registration times on the order of 60 seconds.

\subsection{Direct Measurements of Cup Alignment from Postoperative CT}

To define the anatomic reference system of the pelvis, the anterior pelvic plane (APP) was defined in the CT-scan space. The two anterior superior iliac spines and the pubic tubercles, were identified manually by inspecting the three orthogonal cross sections of the CT scan. Because of the presence of the THR implants in the scan and the resulting imaging artifact, it was not possible to segment these landmarks automatically. Images were then segmented with an intensity threshold set to a level where only the metallic cup appears in the image. This selection was performed in 
order to eliminate the image reconstruction artifacts and allow generation of an artifact-free, segmented surface model of the acetabular cup. Renderings of a CAD 3D model of the cup were then generated and displayed on the computer screen, and overlaid onto CT cross sections and the segmented surface model of the cup. This model was manipulated until the best possible match was found in all views. The final orientation was recorded as cup abduction and version in the anatomic reference system of the pelvis.

The differences between 2D/3D registration and direct CT-based measurements for cup abduction and version were defined as abduction and version errors in the 3D anatomic reference frame and used to validate the method. The pelvic orientation was measured with $2 \mathrm{D} / 3 \mathrm{D}$ registration method and reported in terms of pelvic flexion relative to the plane of the $\mathrm{x}$-ray film.
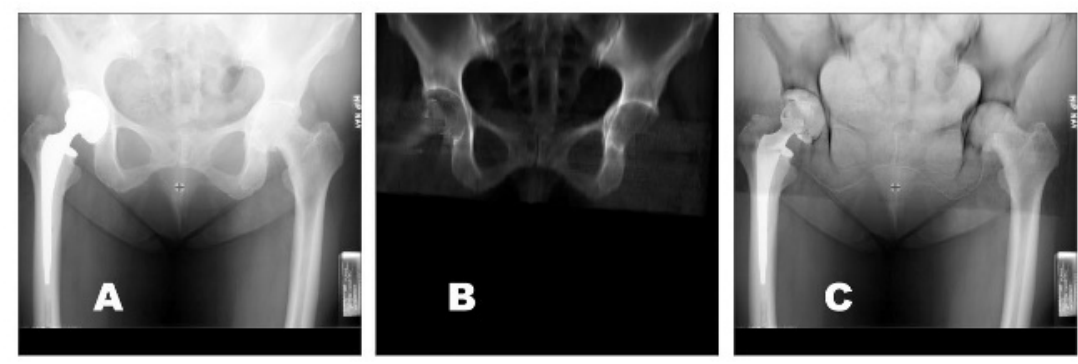

Fig. 2. Registration of the pelvis: (a) real radiograph (b) simulated radiograph corresponding to the solved pose of the CT in the $\mathrm{x}$-ray space (c) real and simulated radiographs overlaid

\subsection{Laboratory Experiment}

The procedure was first tested in the laboratory with a hard plastic phantom pelvis in which an acetabular cup was implanted. The model was mounted on an acrylic sheet in order to increase rigidity and to improve the definition of the anterior pelvic plane. Three small metallic fiducial markers were fitted to the acrylic plate in order to create a well-defined pelvic coordinate system. Two of these fiducials were placed near the anterior iliac spine points. The third fiducial was mounted between the left and right pubic tubercle points. A CT scan, with an in-plane resolution of $0.781 \mathrm{~mm} / \mathrm{pixel}$ and an inter-slice distance of $1.25 \mathrm{~mm}$, was then obtained.

A CT-based Preoperative Planner for THR [4] was used to determine the actual acetabular implant orientation from the CT scan. After measuring the acetabular implant orientation, the implant was digitally removed from the CT scan in order to simulate a preoperative $\mathrm{CT}$ and to make the alignment process more realistic. The acrylic sheet and the fiducials were digitally removed as well. The OptoTrak system (Northern Digital, Inc., Ontario, Canada) was used to verify the Planner cup orientation measurement. The locations of the fiducials and the location and orientation of the acetabular cup were digitized relative to a pelvic tracker using a series of pointer probe collections. The abduction/version of the cup differed from the Planner measurements by less than $0.2^{\circ}$ in abduction and by less than $0.3^{\circ}$ in version. 

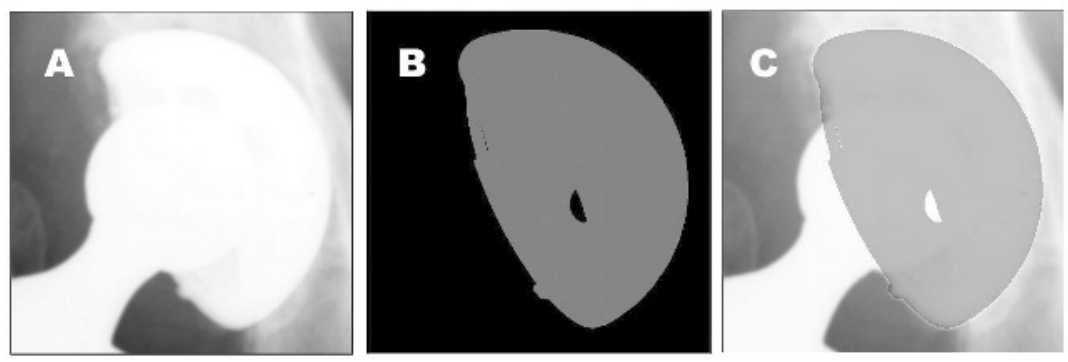

Fig. 3. Registration of the cup: (a) real radiograph (b) simulated projection for the solved pose of the cup (c) the real and simulated images overlaid

Four AP X-rays, one for training and three for testing, were taken of the pelvic assembly using the standard 40" source-to-film distance. A small metallic washer was used to mark the X-ray source position on the film. Three of these X-rays were taken with the pelvis in a near neutral pose and one was taken with a pronounced pelvic flexion of $-11^{\circ}$. The source position ranged from above the bottom of the sacrum to above the top of the sacrum. A group of ten volunteers used $2 \mathrm{D} / 3 \mathrm{D}$ registration software to measure implant pose. The measurements were taken in the following manner. First, the user roughly aligned the CT based synthetic X-ray with the real Xray. The user then instructed the system to perform an automatic CT/radiograph alignment using the procedure outlined in the previous subsection. Finally, the users were able to apply additional manual manipulations and the automatic method until they were satisfied with the match.

\subsection{Clinical Experiment}

This analysis was performed using the postoperative pelvic X-rays and CT scans of 19 patients, who underwent bilateral (but not simultaneous) primary total hip replacement (THR), guided by surgical navigation [2]. The included patients had two preoperative pelvic CT scans, one before each intervention. The second of the two scans imaged the first total hip prosthesis, and this second scan was included in this study as a postoperative CT. There were 11 women and 8 men with a mean age of 61 years (range, 49-82 years). The same uncemented, metal-backed type of acetabular implant was used in all cases. (Trilogy, Zimmer, Warsaw, IN). Antero-posterior (AP) supine pelvic radiographs obtained closest to the date of the second CT scan were used for radiographic measurements.

\section{Results}

\subsection{Experimental Measurements}

For the phantom study, the average error between the $2 \mathrm{D} / 3 \mathrm{D}$ registration measurements and the CT Planner measurements was $-0.05^{\circ}$ in abduction and $-0.37^{\circ}$ in version with a standard deviation of $0.16^{\circ}$ and $0.94^{\circ}$ respectively. The range of 
values for abduction was $-2.49^{\circ}$ to $0.21^{\circ}$ and $-3.10^{\circ}$ to $1.68^{\circ}$ for version. A paired ttest analysis using Microsoft Excel 2000 was performed to determine if there was any significant difference (alpha $>0.017$ using the Bonferroni multiple comparisons correction) in the results based on the X-ray image used. There was no significant difference between any pair of images.

\subsection{Clinical Measurements}

\subsubsection{Direct CT Measurements of Cup Alignment and Pelvis Orientation}

Measured directly from postoperative CT scans of 19 patients, the mean ( \pm standard deviation) $\mathrm{CT}$ cup abduction was $52^{\circ} \pm 5^{\circ}$ and ranged from $43^{\circ}$ to $59^{\circ}$. The mean acetabular cup version was $18^{\circ} \pm 7^{\circ}$ (range $-0.5^{\circ}-29^{\circ}$ ). Pelvis flexion angles in CT scans ranged from $-14^{\circ}$ to $15^{\circ}$, with a $1.6^{\circ}$ mean and a standard deviation of $6.5^{\circ}$. This data range represents the anatomic inter-subject variability of the pelvic flexion angles.

\subsubsection{Cup Position and Pelvic Orientation from AP X-Rays Using CT Matching} The mean ( \pm standard deviation) acetabular cup abduction was $52.9^{\circ} \pm 4.8^{\circ}$, ranging from $46.0^{\circ}$ to $60.1^{\circ}$. The cup version ranged from $3.1^{\circ}$ to $29.7^{\circ}$, with a mean $( \pm$ standard deviation) of $18.7^{\circ} \pm 6.6^{\circ}$. The measurement differences, compared to CT measurements, were $0.4^{\circ} \pm 0.8^{\circ}$ (mean \pm standard deviation) for cup abduction and $0.6^{\circ} \pm 0.79^{\circ}$ (mean \pm standard deviation) for cup version. The individual measurements are shown in Figure 4. The maximum absolute errors were $2.15^{\circ}$ for abduction and $2.03^{\circ}$ for version. The mean pelvic flexion was $3.9^{\circ} \pm 4.9^{\circ}$ ( \pm standard deviation) and its values ranged from $-5.9^{\circ}$ to $11.2^{\circ}$.

\section{Difference in cup orientation measurements}

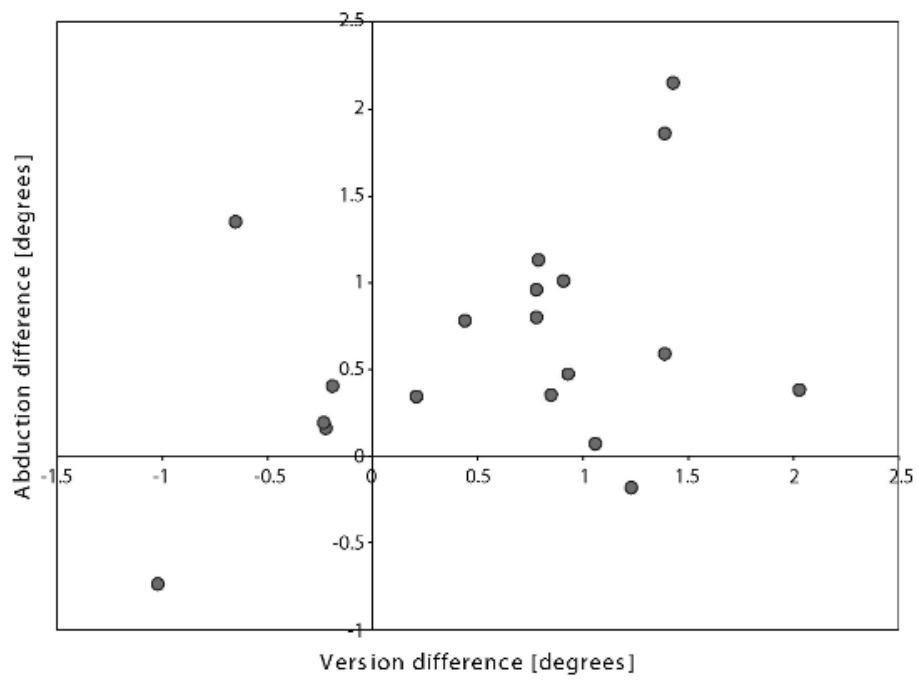

Fig. 4. Differences in measured cup orientation between direct CT measurements and the 2D/3D registration method 


\section{Discussion}

Using 2D/3D registration with the automatic mutual information method, the threedimensional anatomic orientation of the acetabular implant can be determined accurately from a single AP X-ray. Implant version is measured with a slightly lesser accuracy than abduction, but still within the practical range. Version accuracy might be improved by adapting the $2 \mathrm{D} / 3 \mathrm{D}$ registration algorithm to work with multiple $\mathrm{X}$ rays views simultaneously. Tissue noise, scatter noise, and a lower resolution scanning protocol may reduce the accuracy of clinical measurements. The scatter produced by the metallic femoral head may also reduce the accuracy of the postoperative $\mathrm{CT}$ reference measurements.

It is important to stress that the $2 \mathrm{D} / 3 \mathrm{D}$ registration results for cup and pelvic orientation are all referenced anatomically. The difference between 2D/3D values and $\mathrm{CT}$ values for cup orientation were considered as $2 \mathrm{D} / 3 \mathrm{D}$ registration measurement errors. The mean errors ( \pm stdev), $0.4^{\circ} \pm 0.8^{\circ}$ for abduction and $0.6^{\circ} \pm 0.8^{\circ}$. for version are considered to be very good. This is an improvement over previously reported errors for manual matching. Although bilateral scans are used as the best practical ground truth the error between the CT and 2D/3D registration is of the same order of magnitude as the variability in direct CT measurements. Therefore, in the clinical experiment, beyond stating the difference between the two measurement techniques, it is impossible to attribute the error to either of the modalities/measurement techniques. It takes between 1 and 2 minutes to complete both automatic registrations. It can therefore be concluded that the presented method satisfies both accuracy and performance requirements to be used as a routine clinical tool. These measurements require CT data or some other form of 3D imaging. However, the algorithm requires just one pelvic CT scan that could be obtained preoperatively or postoperatively, at any moment in time, and then used for multiple postoperative X-ray measurements throughout the patient's life.

Nishihara and coworkers [5] recently reported a similar methodology. They used, however, a different approach to the CT/X-ray matching process, based on calculating and matching different ratios between pelvic diameters measured on AP X-rays and the 3D pelvic CT surface model. The authors found that the supine pelvic flexion variation of the studied patient group (101 patients) was even higher than our findings, ranging from $-37^{\circ}$ to $30^{\circ}$. The difference is probably due to their larger study population. The sample size in our clinical study was too small to derive any similar conclusions with sufficient significance. We intend to perform a larger clinical study in the future to examine the variability in patients' pelvis position during radiographic examination and its effect on the perceived cup orientation.

\section{References}

1. Blendea S, Eckman K, Jaramaz B, Levison TJ, DiGioia AM III.: Measurements of Acetabular Cup Position and Pelvic Spatial Orientation After THA Using CT / X-ray Matching. Computer Aided Surgery, Volume 10, January 2005, 37-43.

2. DiGioia A, Jaramaz B, Blackwell M, et al.: The Otto Aufranc Award. Image guided navigation system to measure intraoperatively acetabular implant alignment. Clin Orthop 1998, Oct (355); 8-22 
3. Jaramaz B, DiGioia A, Blackwell M, Nikou C: Computer assisted measurement of cup placement in total hip replacement. Clin Orthop 3541(998) 70-81.

4. Nikou C, Jaramaz B, DiGioia AM III, et al.: POP: Preoperative planning and simulation software for total hip replacement surgery. MICCAI 1999, (1999), 868-875.

5. Nishihara S, Sugano N, Nishii T, Ohzono K, Yoshikawa H: Measurements of pelvic flexion angle using three-dimensional computed tomography. Clin Orthop 411 (2003),140-151.

6. Pluim JPW, Maintz, JBA, Viergever MA.: Image registration by maximization of combined mutual information and gradient information. IEEE Trans. Med. Imag., August 2000, Vol. $19,809-814$.

7. Russakoff DB, Tomasi C, Rohlfing T, Maurer CR Jr.: Image Similarity Using Mutual Information of Regions. ECCV 2004, LNCS 3023, 2004, 596-607.

8. Shannon C: A mathematical theory of communication. Bell System Technical Journal, Vol. 27, July 1948, pp. 379-423., 623-656.

9. Viola P, Wells WM.: Alignment by maximization of mutual information. in Proc. 5th Intl. Conf. Computer Vision, Cambridge, MA, June 1995, 16-23. 